\title{
15
}

\section{CSO Discharge Reporting using a Continuous Modeling Approach}

\author{
Srini Vallabhaneni, Bob Masbaum, Carl Chan and Mario \\ Mazza
}

Many combined sewer communities in the United States require reporting combined sewer overflow (CSO) discharges to a state regulatory agency on a regular basis to satisfy National Pollution Discharge Elimination System (NPDES) permit requirements. Typically, these requirements include gathering discharge data using automatic flow meters in each outfall and develop the CSO discharge monitoring reports (DMR).

Reliable and consistent approaches are critical in preparing ongoing CSO discharge reports to satisfy NPDES permit requirements and to track longterm trends in discharges. Outfall flow monitoring presents many challenges and relying upon the outfall monitoring equipment alone to estimate overflow volumes may lead to inconsistent and unreliable information. The DMR data reported based entirely on monitoring data may add more complexity (in addition to the rainfall variations, system changes, etc.) when assessing the efficacy of implemented CSO controls. Continuous simulations with the interceptor hydraulic model supported by CSO activation monitoring data is the most cost-effective, reliable, and consistent method for generating continuous CSO discharge data to meet permit requirements.

This chapter will present a case study based on extensive experience in preparing CSO DMRs for the City of Indianapolis, Indiana by applying the calibrated sewer system hydraulic model on a continuous basis. Continuous

Vallabhaneni, S., B. Masbaum, C.C. Chan and M. Mazza. 2007. "CSO Discharge Reporting using a Continuous Modeling Approach." Journal of Water Management Modeling R227-15. doi: 10.14796/JWMM.R227-15.

(C) CHI 2007 www.chijournal.org ISSN: 2292-6062 (Formerly in Contemporary Modeling of Urban Water Systems. ISBN: 0-9736716-3-7) 
modeling techniques and protocols developed for a complex Indianapolis sewer system are presented along with important lessons learned.

\subsection{Introduction}

The City of Indianapolis Department of Public Works serves the wastewater needs of Marion County in central Indiana. Population in the service area is approximately 880,000 , based on 2000 Census data. Approximately 400 square miles $\left(1036 \mathrm{~km}^{2}\right)$ of service area is served by both combined and separate sewer systems. Over 30 combined and sanitary interceptors convey wastewater flows to two advanced wastewater treatment plants (AWTP).

The combined sewer area covers approximately 55.5 square miles $\left(144 \mathrm{~km}^{2}\right)$, is located primarily in the older sections of the city, and contains 132 combined sewer outfalls. Figure 15.1 depicts the Marion County service area and combined sewer area. Combined sewers overflow when the wastewater and rainfall runoff exceeds the capacity of the regulators or interceptor sewers. Interceptors are typically smaller than combined sewers and reach capacity much more frequently. Under normal conditions, regulators divert these combined flows into interceptor sewers for conveyance to one of the two treatment facilities.

Typically, a regulator consists of a dam or weir in the combined sewer pipe that conveys dry-weather flows to the interceptor, which allows overflows to the receiving stream through an outfall pipe. Figure 15.2 shows a typical layout of a CSO regulator in the Indianapolis system. There are 169 regulator structures in the Indianapolis combined sewer system. Some CSO outfalls serve more than one regulator.

CSOs are regulated under the Clean Water Act and its National Pollutant Discharge Elimination System (NPDES) program, which permits and regulates wastewater discharges. In Indiana, NPDES permits are issued by the Indiana Department of Environmental Management (IDEM), under a delegation agreement with the U.S. Environmental Protection Agency (EPA). Both state and federal regulators have authority to enforce these permits. IDEM issued NPDES permits for both the Belmont and Southport AWTPs in October 2001. Compliance with the permits requires that the city must report, at 6-month intervals, CSO volume and duration from each outfall on an ongoing basis based upon a hydraulic model of the sewer system. In addition, the city must report CSO activation data on monthly basis, based on automatic activation monitors. 


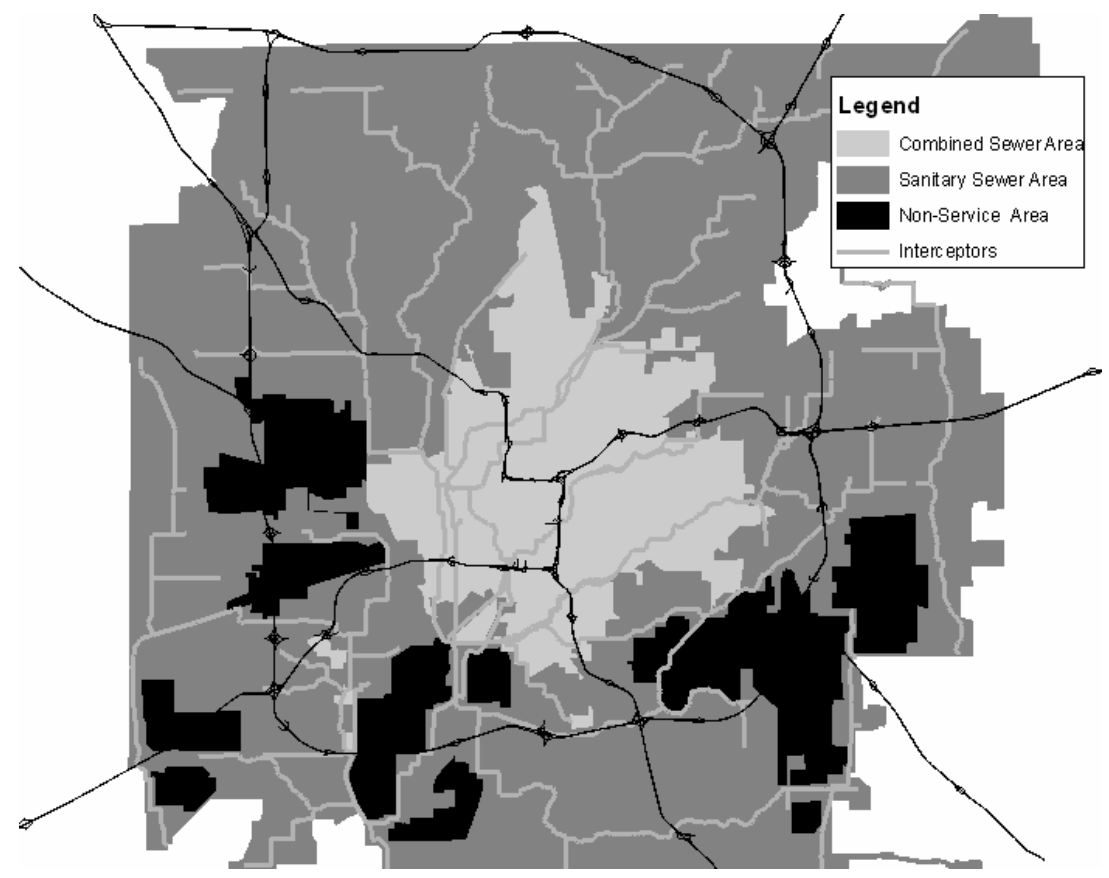

Figure 15.1 Marion County service area and combined sewer area.

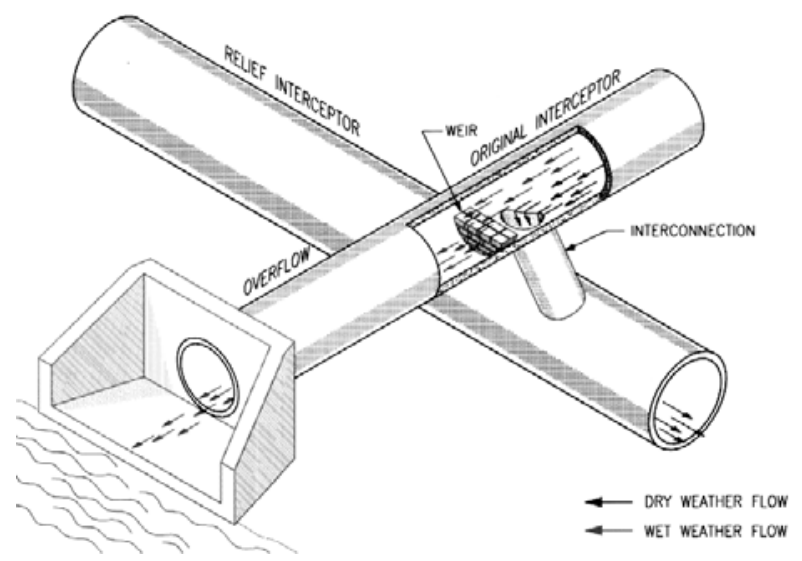

Figure 15.2 Typical Indianapolis CSO regulator. 
The primary purpose of the model-based DMR is to document estimates of the CSO discharges from the city's combined system on an ongoing basis. The city's hydraulic model is applied for each 6-month period using continuous precipitation records to estimate $\mathrm{CSO}$ volume and duration. The CSO database compiled from the DMRs is also expected to assist the city and IDEM in tracking trends in CSO discharge characteristics as the city makes progress in its efforts to reduce CSOs. The CSO discharge characteristics reported include volume and duration on a daily basis. As part of its CSO planning efforts, the city has been generating the 6-month DMRs since 1999, using the hydraulic model of the sewer system. Data generated for these DMRs has been used extensively for CSO control efforts and in support of sewer system management functions.

\subsection{Hydraulic Model Description}

The city has developed and calibrated a computer model of the interceptor sewer and CSOs using EPA's Storm Water Management Model (SWMM). This hydraulic model is a key element in developing the CSO Long Term Control Plan (LTCP). The model includes representation of all 132 CSO outfalls in the Indianapolis combined sewer system and is capable of producing CSO estimates.

The hydraulic model development started in 1992 and has evolved over time. At the time of this writing, the model resides in a version of SWMM 4.4 that is customized for Indianapolis system needs. The city uses the RUNOFF and EXTRAN blocks of SWMM to model the combined sewer system hydraulics. RUNOFF is used to calculate the rates of stormwater runoff entering the combined sewer system; EXTRAN is then used to route the stormwater and sewage flows through the system. Since 1992, the model has been updated to reflect new sewer system data and operational changes. The model was first calibrated in 1996 and then recalibrated in 2002 using extensive flow monitoring data. EPA performed detailed reviews of the 2002 model recalibration effort and approved the model for CSO LTCP development in June 2002. Currently, the Indianapolis interceptor sewer system hydraulic model includes approximately 3,000 nodes, 169 CSO regulators, and $132 \mathrm{CSOs}$. The modeled pipe size range between 24 in. $(61 \mathrm{~cm})$ to $154 \mathrm{in} .(391 \mathrm{~cm})$. The length of modeled sewer is approximately 25 miles $(40 \mathrm{~km})$.At the time of this writing, the city is in the process of converting its hydraulic model to the current version of SWMM5. 
Additionally, the city is systematically expanding the model into each combined sewer basin upstream of the CSO regulators and the model is projected to exceed a total of 10,000 nodes by 2008 . The estimated sewer length in the model will be approximately 50 miles $(80.5 \mathrm{~km})$.

The model-based DMRs are prepared within six months following each 6-month reporting period. For example, the DMR for the reporting period January through June 2005 is generated by December 2005. The City of Indianapolis has been using the hydraulic model on a continuous basis to generate the CSO volume and duration for every 6-month period since 1999. The city has gained many insights into continuous modeling and has developed efficient methods to address the challenges that come with it. As a result, the protocol for model application for the 6-month DMRs has been developed and refined with each successive application of the model. The authors believe that protocols and insights developed for continuous modeling in Indianapolis can benefit complex sewer systems in other cities. The following outlines the steps in performing the 6-month DMR and notes some worthy insights.

This protocol is divided into four sections: Model Data Collection, Model Update, Model Simulations, and Model Results Processing.

\subsubsection{Model Data Collection and Review}

This key step involves collecting data on system improvements and modifications made since the previous DMR period, including the following:

- Collect and review any new operational data and verify any changes in the operational strategy both in the collection system and at the Advanced Wastewater Treatment Plants (AWTP).

- Collect and review information on all sewer system improvement projects that were completed and put into operation since generation of the previous DMR.

- Review the sewer system routine operation and maintenance activities (e.g., sewer cleaning) and assess the need for model refinements.

- Collect and review the rain gauge and radar processed rainfall data for the 6-month DMR period.

- Prepare the rainfall data in the format necessary for the SWMM model. 
- Collect and review CSO activation data at each CSO outfall to use for continued validation of the hydraulic model results.

- Collect any other operating data such as the in-system realtime control gate operation and prepare for model application.

Besides the information on recent sewer system changes or capital improvement projects, sewer system operational data and radar-rainfall data are the two most important inputs to the recurring long-term continuous simulations.

\section{Operating Data}

Sewer operations such as the flow diversion control gate, pump station, inflatable dam, and other real-time control gate operations can be input into the EXTRAN model in two ways: using standard operating procedures (SOP) or actual operating data (i.e. time series of gate/pump operations). These operations have a direct impact on the modeling results and estimates of CSO volume and duration. For example, in the City of Indianapolis, a critical flow diversion structure with real-time gate controls regulates the flow to the two AWTPs, and consequently has a direct impact on CSO discharges at the three largest CSOs in the system.

An SOP is in place to assist the operators in managing sewer system controls under normal circumstances. However, at times, such as during an extreme rainfall event or other special circumstances, operators may override the SOP and operate sewer system controls to yield optimum levels of wastewater treatment and system overflows. Operator experience and the data collected to date indicate that the actual operation often deviates from the SOPs. To report CSO discharge volumes accurately, the hydraulic model simulations must consider actual operation data rather than the SOP. Operation data for the 6-month DMR period is extensive and requires considerable time to review and process before incorporating into the EXTRAN model using the F3/F4 features. (In SWMM 5, "CONTROL" feature allows simulating the system hydraulics using the actual operating data).

\section{Spatially Varied Rainfall}

For a model that represents a large metropolitan area such as Indianapolis, it is critical to have spatially varied rainfall in order to produce accurate results on an outfall-by-outfall basis. Rainfall is the most sensitive input parameter 
in any hydraulic model simulations under wet weather flow conditions. The reliability of rainfall data directly affects the accuracy of estimating CSO discharge volume in the hydraulic model (Koran et al. 2002). It has been demonstrated that spatially varied rainfall, such as rain gauge calibrated radar-rainfall data, can improve the accuracy of the model results (Burgess et al. 1997 and Koran et al. 2002). The City of Indianapolis has adopted radar-rainfall technology into their modeling efforts since 1995. Steps in successful integration of radar-rainfall technology can be found in the 2004 CHI conference monograph-12. (Vallabhaneni et al., 2004).

\subsubsection{Perform Model Updates}

This step involves incorporating any sewer system changes or changes in operational strategies made since the last DMR model application. Capital improvement projects or CSO LTCP-related projects completed since the last DMR period are incorporated into the hydraulic model. For example, if a CSO storage facility is completed and put into operation during the DMR period, the model is modified to include the storage facility and appropriate piping changes. Results of any sewer system changes such as modifications made to flow regulating gate operations upstream of the AWTPs, and maintenance activities such as sewer cleaning, are also incorporated.

\subsubsection{Perform Continuous Model Simulations}

The next step is performing the model simulation using rainfall input for the 6-month period and de-bugging the model as necessary. Two main challenges in performing continuous model simulations are the model runtime and model de-bugging.

\section{Model Run Time}

The City of Indianapolis hydraulic interceptor model contains over 3,000 nodes, including 169 regulator structures, and actual continuous operating data for the control gates. A 1-s time-step was used in the EXTRAN model. Even with today's advanced computing technology, on average, it would take 20-36 hours to complete a 6-month continuous simulation for Indianapolis' complex hydraulic interceptor model at the 1-s time step, using an Intel Pentium 4, $3.4 \mathrm{GHz}$ machine. The model run-time is directly related to the specific distribution of rainfall during the simulation period and the stability of the EXTRAN model's solution algorithm. The rainfall 
distributions cannot be modified and therefore, in order to shorten the model run-time, it is important to de-bug the model such that it will run smoothly through the 6-month continuous simulation.

The most popular method to shorten model run-time is to increase the EXTRAN time step. This practice can reduce model run-time during dry weather conditions. However, during rainfall events, it requires more iterations, and hence more computation time, to fully solve the flow equations (i.e. the St. Venant equations). Based on experience in performing continuous simulations for DMRs since 1999, it has been determined that increasing the EXTRAN time step cannot always help to solve the model run-time challenge. It has been concluded that the additional time it takes to fully converge the model during wet-weather periods is far greater than the time saved in increasing the EXTRAN time step.

This also suggests that the most effective way to reduce model simulation run-time is to reduce the number of excessive iterations required to successfully converge the model computations during wet-weather conditions. A two-step approach has been found effective:

1. determining an optimum EXTRAN model time step; and

2. in-depth model de-bugging.

An EXTRAN model time step of 1-second has resulted in satisfactory model stability. Using this time step with in-depth model de-bugging (see below) helped reduce the number of excessive iterations to converge the model and therefore, reduced the overall model simulation time. Once the model conversion to the latest version of SWMM5 is completed, relatively faster model run times are anticipated due to code improvements.

\section{Model De-Bugging}

Troubleshooting, or "de-bugging" the model for continuous simulation can be time consuming and frustrating, and requires a systematic approach to maximize efficiency. If the model is not adequately de-bugged and prepared well for continuous simulation, it may result in significant inefficiencies in project execution and potential inaccuracies in model results.

Experience indicates that the model de-bugging effort is a function of the specific rainfall distribution in the 6-month DMR period of interest. Typically, rainfall events with high intensity result in model instability. However, it has been observed that instability in EXTRAN simulation can occur during back-to-back rainfall events with moderate intensity. In addition, specific antecedent moisture conditions can also influence model stability during an event with moderate intensity. Therefore, a systematic 
approach is required to efficiently de-bug the model and ensure a smooth continuous simulation.

The first step in the model de-bugging efforts is developing a thorough knowledge of the rainfall distributions during the 6-month DMR period. The following outlines the review of rainfall data:

- perform detailed review of rainfall distributions in the 6-month period;

- select the top three to five intense single events;

- select two to three distinct back-to-back rainfall events; and

- select a two- to three-week period with frequent rainfall events and with the highest cumulative rainfall.

The model de-bugging is performed in three steps under three types of rainfall conditions during the 6-month period:

Step 1

Apply the model using single event simulation using a minimum of three high intensity rainfall events, as suggested below. The purpose of this step is to ensure model stability during the peak wet-weather events during the continuous simulation period.

- First single event with the highest rainfall intensity during the continuous simulation period.

- Second single event with the highest rainfall volume during the continuous simulation period.

- Third single event with the second-highest rainfall volume during the continuous simulation period.

Step 2

Perform model simulations with a minimum of two back-to-back rainfall events (defined as two rainfall events that occur within $48 \mathrm{~h}$ ). The purpose of this step is to ensure model stability during the combination of large to moderate rainfall events and wet antecedent moisture conditions:

First back-to-back event with highest rainfall volume during the continuous simulation period.

- Second back-to-back event with a balanced distribution of high rainfall volume between two events.

Step 3

Perform short-term continuous simulations using selected two- to threeweek period. The purpose of this step is to ensure model stability in a continuous simulation situation. Modelers should identify a two to three 
week period with the largest precipitation record during the continuous simulation period.

This three-step approach can effectively "stress test" the model under different rainfall distributions during the 6-month period. Once the model is stabilized under these conditions, it is less likely to experience instability during the 6-month continuous simulation.

After completing these model de-bugging procedures, a 6-month simulation can be performed. It should be expected that the need for additional model de-bugging may arise. Once the 6-month simulation is completed successfully, the model results are reviewed and validated. The CSO activation data recorded by automatic flow activation equipment from each outfall for the corresponding 6-month period is used to validate the CSO activation data based on the model simulations. This validation process identifies any limitations in the rainfall data, model representation of the new sewer improvements, model convergence, and inaccuracies in the CSO activation data recorded using the automated equipment. Once this quality assurance process is complete, the model results are ready to be processed for reporting. Any future long-range improvements such as additional modeling details flow monitoring, and recalibration that may further enhance the model accuracy are documented.

\subsubsection{DMR Preparation}

The 6-month continuous model simulation full output file, which is very large (in the order of 10-12 gigabytes), may be unreadable because of the computer limitations. A subset of pipe-specific model results, which can be created using the EXTRAN B9 feature, is used to report the CSO volume discharge. This feature allows users to have complete output control. This special output is then post-processed using a customized computer tool called CSO Reporter. This tool, developed for DMR purposes, will process the large amount of data in the model output file efficiently and produce data tables for the CSO DMR.

The CSO Reporter tool will process the EXTRAN model output files as well as the rainfall input data. After post-processing, this tool can produce daily rainfall volume, daily runoff, and CSO discharge volume and duration data for each outfall. In addition, this tool can track the CSO discharge information by each receiving watershed within the system. For example, a portion of actual DMR data table is presented in Table 15.1. For each CSO, monthly total discharge, maximum daily CSO discharge and the date of maximum discharges for each month are noted. The DMR tables also 
include daily rainfall data. The CSO basin-specific rainfall data is derived from the city's radar-rainfall system. In addition to presenting the information by CSO, the DMR provides a daily summary of discharges, grouped by various watersheds within the system.

Table 15.1 Sample CSO DMR data table.

INDIANAPOLIS CSO CONTROL PROGRAM

NPDES \#IN0023183

July $2003 \quad$ Page 1 of 26

ESIMATED CSO DURATION AND OVERFLOW VOLUME

\begin{tabular}{|c|c|c|c|c|c|c|c|c|c|c|}
\hline \multirow[b]{3}{*}{ Day } & \multicolumn{4}{|c|}{ CSO049 } & \multicolumn{4}{|c|}{ CSO050 } & \multirow{3}{*}{$\begin{array}{c}\text { TOTAL } \\
\text { RAINFALL } \\
\text { VOLUME } \\
\text { (MG) }\end{array}$} & \multirow{3}{*}{$\begin{array}{c}\text { TOTAL } \\
\text { OVERFLOW } \\
\text { VOLUME } \\
\text { (MG) }\end{array}$} \\
\hline & \multicolumn{2}{|c|}{ Rainfall } & \multicolumn{2}{|c|}{ Overflow } & \multicolumn{2}{|c|}{ Rainfall } & \multicolumn{2}{|c|}{ Overflow } & & \\
\hline & $\begin{array}{c}\text { TR } \\
\text { (in.) } \\
\end{array}$ & $\begin{array}{c}\text { TR } \\
\text { (MG) }\end{array}$ & $\begin{array}{c}\text { Vol } \\
\text { (MG) }\end{array}$ & $\begin{array}{l}\text { Dur } \\
\text { (hr) }\end{array}$ & $\begin{array}{c}\text { TR } \\
\text { (in.) }\end{array}$ & $\begin{array}{c}\text { TR } \\
\text { (MG) }\end{array}$ & $\begin{array}{c}\text { Vol } \\
\text { (MG) }\end{array}$ & $\begin{array}{l}\text { Dur } \\
\text { (hr) }\end{array}$ & & \\
\hline 1 & 0.00 & 0.00 & 0.00 & 0.0 & 0.00 & 0.00 & 0.00 & 0.00 & 0.00 & \begin{tabular}{|l|l}
0.00 \\
\end{tabular} \\
\hline 2 & 0.00 & 0.00 & 0.00 & 0.0 & 0.00 & 0.00 & 0.00 & 0.00 & 0.00 & 0.00 \\
\hline 3 & 0.00 & 0.00 & 0.00 & 0.0 & 0.00 & 0.00 & 0.00 & 0.00 & 0.00 & 0.00 \\
\hline 4 & 0.40 & 1.03 & 0.70 & 2.9 & 0.46 & 1.18 & 1.94 & 2.50 & 2.21 & 2.64 \\
\hline 5 & 3.78 & 9.73 & 7.54 & 15.6 & 3.62 & 9.32 & 23.57 & 13.30 & 19.06 & 31.11 \\
\hline 6 & 0.00 & 0.00 & 0.00 & 0.0 & 0.00 & 0.00 & 0.00 & 0.00 & 0.00 & 0.00 \\
\hline 7 & 0.00 & 0.00 & 0.00 & 0.0 & 0.00 & 0.00 & 0.00 & 0.00 & 0.00 & 0.00 \\
\hline 8 & 0.67 & 1.73 & 1.04 & 3.2 & 0.64 & 1.65 & 3.26 & 2.80 & 3.37 & 4.30 \\
\hline 9 & 0.66 & 1.70 & 0.66 & 6.3 & 0.76 & 1.96 & 1.95 & 5.30 & 3.66 & 2.61 \\
\hline 10 & 0.31 & 0.80 & 0.59 & 4.3 & 0.32 & 0.82 & 1.78 & 3.80 & 1.62 & 2.37 \\
\hline 11 & 0.19 & 0.49 & 0.09 & 1.3 & 0.24 & 0.62 & 0.33 & 1.80 & 1.11 & 0.42 \\
\hline 12 & 0.00 & 0.00 & 0.00 & 0.0 & 0.00 & 0.00 & 0.00 & 0.00 & 0.00 & 0.00 \\
\hline 13 & 0.00 & 0.00 & 0.00 & 0.0 & 0.00 & 0.00 & 0.00 & 0.00 & 0.00 & 0.00 \\
\hline 14 & 0.00 & 0.00 & 0.00 & 0.0 & 0.00 & 0.00 & 0.00 & 0.00 & 0.00 & 0.00 \\
\hline 15 & 0.25 & 0.64 & 0.08 & 1.3 & 0.22 & 0.57 & 0.07 & 1.10 & 1.21 & 0.15 \\
\hline 16 & 0.00 & 0.00 & 0.00 & 0.0 & 0.00 & 0.00 & 0.00 & 0.00 & 0.00 & 0.00 \\
\hline 17 & 0.01 & 0.03 & 0.00 & 0.0 & 0.02 & 0.05 & 0.00 & 0.00 & 0.08 & 0.00 \\
\hline 18 & 0.34 & 0.88 & 0.24 & 3.3 & 0.33 & 0.85 & 0.54 & 2.50 & 1.73 & 0.78 \\
\hline 19 & 0.00 & 0.00 & 0.00 & 0.0 & 0.00 & 0.00 & 0.00 & 0.00 & 0.00 & 0.00 \\
\hline 20 & 0.00 & 0.00 & 0.00 & 0.0 & 0.00 & 0.00 & 0.00 & 0.00 & 0.00 & 0.00 \\
\hline 21 & 1.06 & 2.73 & 1.79 & 6.8 & 0.93 & 2.39 & 4.50 & 6.30 & 5.12 & 6.29 \\
\hline 22 & 0.00 & 0.00 & 0.00 & 0.0 & 0.00 & 0.00 & 0.00 & 0.00 & 0.00 & 0.00 \\
\hline 23 & 0.00 & 0.00 & 0.00 & 0.0 & 0.00 & 0.00 & 0.00 & 0.00 & 0.00 & 0.00 \\
\hline 24 & 0.00 & 0.00 & 0.00 & 0.0 & 0.00 & 0.00 & 0.00 & 0.00 & 0.00 & 0.00 \\
\hline 25 & 0.00 & 0.00 & 0.00 & 0.0 & 0.00 & 0.00 & 0.00 & 0.00 & 0.00 & 0.00 \\
\hline 26 & 0.00 & 0.00 & 0.00 & 0.0 & 0.00 & 0.00 & 0.00 & 0.00 & 0.00 & 0.00 \\
\hline 27 & 0.28 & 0.72 & 0.36 & 2.6 & 0.40 & 1.03 & 1.45 & 2.50 & 1.75 & 1.81 \\
\hline 28 & 0.00 & 0.00 & 0.00 & 0.0 & 0.00 & 0.00 & 0.00 & 0.00 & 0.00 & 0.00 \\
\hline 29 & 0.00 & 0.00 & 0.00 & 0.0 & 0.00 & 0.00 & 0.00 & 0.00 & 0.00 & 0.00 \\
\hline 30 & 0.00 & 0.00 & 0.00 & 0.0 & 0.00 & 0.00 & 0.00 & 0.00 & 0.00 & 0.00 \\
\hline 31 & 0.00 & 0.00 & 0.00 & 0.0 & 0.00 & 0.00 & 0.00 & 0.00 & 0.00 & 0.00 \\
\hline TOTAL & 7.95 & 20.47 & 13.09 & 47.60 & 7.94 & 20.45 & 39.39 & 41.90 & 40.92 & 52.48 \\
\hline
\end{tabular}

TR - Total Rainfall

Vol - Volume

Dur - Duration

\subsection{Application of DMR Data}

The primary purpose of the DMR is to meet NPDES permit requirements. However, the long-term CSO DMR data can provide a sound and consistent means to evaluate overflow characteristics in relation to precipitation conditions and assess the performance of CSO LTCP projects. This data will 
also help to evaluate the average annual system performance and to measure against the target level of CSO control for the constructed projects.
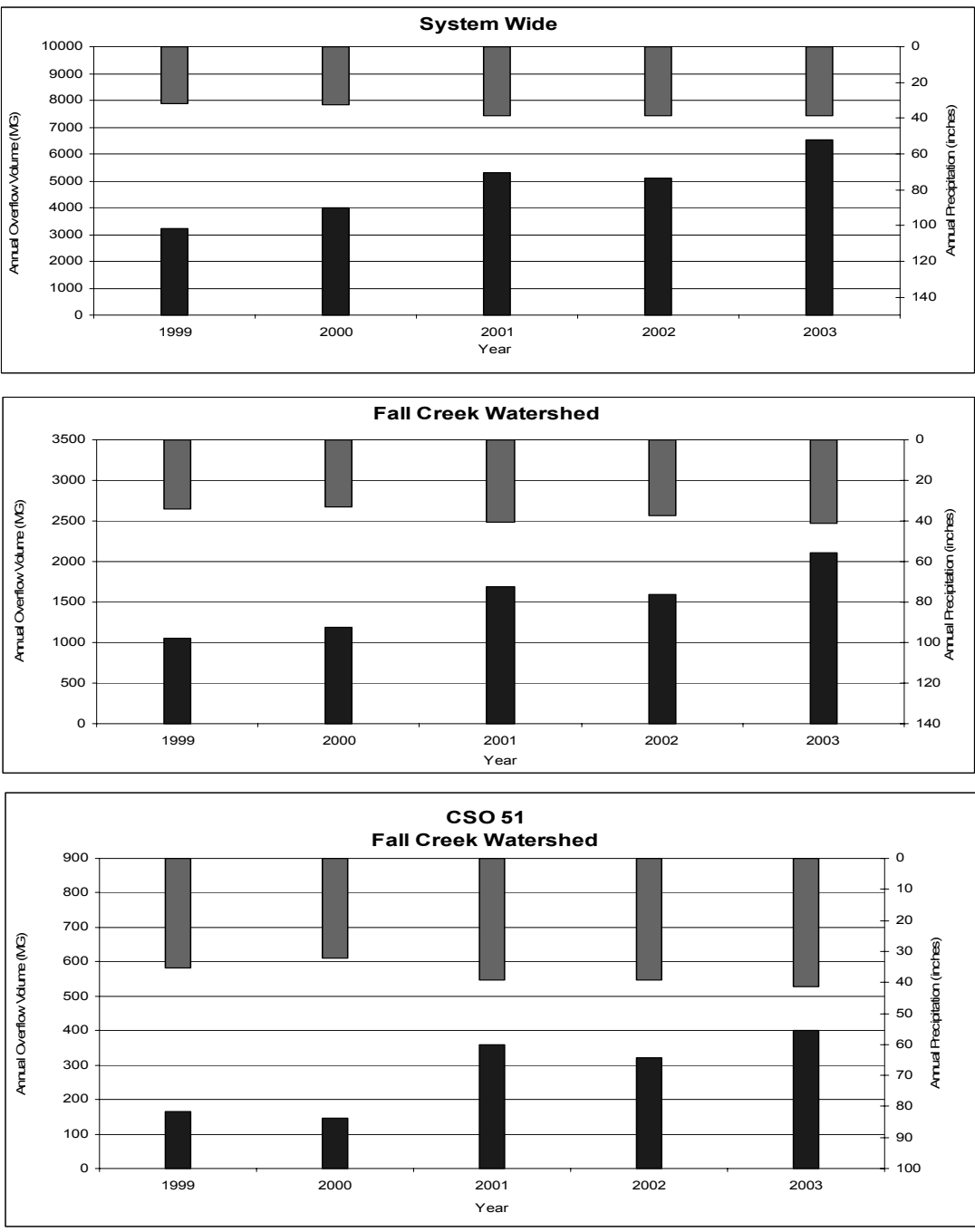

Annual Precipitation

Annual Overflow Volume

Figure 15.3 Sample CSO discharge data and tracking 
As part of CSO planning, the city has been generating the 6-month DMRs since 1999. Data generated for these has been used for CSO control efforts and in support of sewer system management functions. The CSO discharge volume and frequency database has been growing with every 6-month DMR that the city produces. This provides valuable insights on changes in the sewer system wet-weather response in relation to precipitation patterns and system improvements to control CSOs. As the city moves forward with the implementation of LTCP projects, the CSO discharge database can be effectively used to demonstrate the downward trends of system-wide CSO discharges over time.

An example of how the CSO data will be tracked and analyzed is shown in Figure 15.3, which shows the 5-y period (1999-2003) of detailed CSO DMR estimates.As the city is in the initial stages of implementing significant CSO control projects, the CSO discharge data for these five years establishes a baseline condition, which can be used to assess the trends of $\mathrm{CSO}$ volumes and frequencies in the future as the city implements the longterm control plan projects.

\subsection{Conclusions}

Reliable and consistent approaches are critical in preparing ongoing CSO discharge monitoring reports (DMR) to satisfy NPDES permit requirements and to track long-term trends in discharges. Hydraulic model-based 6-month CSO DMRs have been found to be effective in the City of Indianapolis since 1999. Indianapolis has gained many insights in continuous modeling and efficient methods to address the challenges that come with it. As a result, the protocol for model application for the 6-month DMR has been developed and refined with each successive application of the model.

Continuous modeling for complex sewer systems is practical with current computing technology. However, methodical approaches should be developed to realize efficient model execution and thereby costeffectiveness; also data management/processing tools must be obtained and developed to process the large model output. Efficient model simulations and data processing is expected to keep the cost for continuous modeling affordable, and justifies its use to support important decision-making related to expensive sewer system wet-weather overflow control programs.

The existing system performance and the efficacy of CSO LTCP projects must be evaluated on an average annual basis. Wide variations are observed 
in year-to-year precipitation patterns and associated CSO discharges from the combined sewer systems. Long-term CSO DMR data will provide a sound and consistent approach in evaluating overflow characteristics in relation to precipitation conditions and assessing the performance and effectiveness of the CSO LTCP projects. This long-term data is critical to evaluate the average annual performance against the target level of CSO control for the constructed projects. The authors believe that protocols and insights developed for continuous modeling in Indianapolis can benefit other complex sewer systems elsewhere.

\section{Acknowledgments}

The authors thank Carlton Ray of the Indianapolis Department of Public Works for his support in developing model-based CSO discharge monitoring reports since 1999. In addition, the authors thank Daniel Vicari and Animesh Irkulla of CDM for their efforts in data collection and coordination.

\section{References}

Belmont AWT Plan NPDES Permit Number IN 0023183

Burgess, E.H., Stevens, P.L., George, T.D., Vallabhaneni S., Use of Radar ImageEnhanced Precipitation Data in Combined Sewer Overflow Modeling. Water Environment Federation, 70th Annual Conference \& Exposition, Chicago, IL. October 18-22, 1997.

City of Indianapolis Department of Public Works (August 2003), Hydraulic Model Calibration and Verification Report, NPDES Compliance

City of Indianapolis Department of Public Works, Clean Stream Program, Stream Line (Fall 2004)

Huber, W. and Dickinson, R. 1992. Stormwater Management Model, Version 4: User's Manual, Environmental Research Laboratory, Office of Research and Development, U.S. Environmental Protection Agency, Athens, Georgia.

Koran, J., Vallabhaneni, S., Gatterdam, M., Moisio, S., and Burgess, E. Application of Radar-Rainfall Technology in Combined Sewer Model Development and Calibration. Water Environment Federation, 75th Annual Conference \& Exposition, Chicago, IL. October 2002.

Roesner, L., Aldrich, J. and Dickinson, R. 1992. Stormwater Management Model, Version 4: User's Manual, Environmental Research Laboratory, Office of Research and Development, U.S. Environmental Protection Agency, Athens, Georgia.

Vallabhaneni, S., B. Vieux and T. Meeneghan. 2004. "Radar-Rainfall Technology Integration into Hydrologic and Hydraulic Modeling Projects." Journal of Water Management Modeling R220-02. doi: 10.14796/JWMM.R220-02. 